\title{
Cholesterol Metabolism as a Potential Therapeutic Target and a Prognostic Biomarker for Cancer Immunotherapy
}

\author{
Huixian Zhang ${ }^{1-3}$ \\ Wencheng Zhao ${ }^{1,2}$ \\ Xingya $\mathrm{Li}^{3}$ \\ Yayi $\mathrm{He} \mathbb{D}^{1,2}$
}

'Department of Medical Oncology, Shanghai Pulmonary Hospital, Tongj University Medical School Cancer Institute, Tongji University School of Medicine, Shanghai, 200433, People's Republic of China; ${ }^{2}$ Tongji University, Shanghai, 200433, People's Republic of China; ${ }^{3}$ Department of Medical Oncology, The First Affiliated Hospital of Zhengzhou University, Zhengzhou City, Henan Province, 450052, People's Republic of China
Correspondence: Yayi He Department of Medical Oncology, Shanghai Pulmonary Hospital, Tongji University Medical School Cancer Institute, Tongji University School of Medicine, Shanghai, 200433, People's Republic of China

Tel +86-|38I8828623

Email225060।@qq.com

Xingya Li

Department of Medical Oncology, The First Affiliated Hospital of Zhengzhou University, Zhengzhou City, Henan

Province, 450052

Tel +86-13838253946

Email fcclixyl@zzu.edu.cn

\begin{abstract}
Checkpoint-based immunotherapies, such as programmed cell death-1 (PD-1)/ programmed cell death ligand-1 (PD-L1) inhibitors, have shown promising clinical outcomes in many types of cancers. Unfortunately, the response rate of immune checkpoint inhibitors is low. It is very important to discover novel therapeutic targets and prognostic biomarkers. Cholesterol metabolism has been demonstrated to be related to the occurrence and development of a variety of tumors and may provide a new breakthrough in the development of immunotherapy. First of all, cholesterol metabolism in the tumor microenvironment affects the function of tumor-infiltrating immune cells. In addition, intracellular cholesterol homeostasis is an important regulator of immune cell function. Furthermore, drugs that act on cholesterol metabolism affect the efficacy of immunotherapy. What is more, peripheral blood cholesterol level can be a biomarker to predict the efficacy of immunotherapy. In this review, we aimed to explore the potential role of cholesterol metabolism on immunotherapy. By summarizing the major findings of recent preclinical and clinical studies on cholesterol metabolism in immunotherapy, we suggested that cholesterol metabolism could be a potential therapeutic target and a prognostic biomarker for immunotherapy.
\end{abstract}

Keywords: cholesterol metabolism, immunotherapy, immune cell, therapeutic target, biomarker

\section{Introduction}

Immunotherapy for tumors, which has recently achieved clinical success, is revolutionizing cancer treatment. ${ }^{1}$ Checkpoint-based immunotherapies are agents that target the inhibitory receptors expressed on activated T cells. ${ }^{2-4}$ Immune checkpoint inhibitors (ICIs) take effect by overcoming or alleviating tumor-induced immunosuppression, thereby releasing inhibitory T-cell-mediated antitumor responses., 5 Programmed death-1 (PD-1)/programmed death ligand-1 (PD-L1) inhibitors have been approved as a systematic treatment for various types of cancers, such as melanoma, non-small cell lung cancer (NSCLC), hepatocellular carcinoma, renal cell carcinoma and so on. ${ }^{7-10}$ Although immunotherapy has shown the application potential in a variety of malignancies, a relatively small proportion of patients have so far benefited from ICIs. ${ }^{11,12}$ Thus, the discovery of more effective immunotherapy targets and the identification of predictive biomarkers for immunotherapy become urgently warranted.

Cholesterol, a subtype of lipids, plays an important role in cell homeostasis, from the basic components that maintain the integrity and stability of cell membranes to the precursors of different forms of important sterols such as vitamins and 
hormones. ${ }^{13,14}$ Both clinical and experimental studies support the link between changes in cholesterol metabolism and cancer development. ${ }^{15-17}$ On the one hand, elevated serum cholesterol levels have been reported to increase the incidence rate of colorectal, prostate and other cancers. ${ }^{18}$ Considering the concomitant relationship between obesity and hyperlipidemia, a prospective study investigating the association between prediagnosis blood lipid concentrations and breast cancer risk reported that serum cholesterol levels did correlate with breast cancer risk regardless of BMI. ${ }^{19}$ On the other hand, accumulated reports have shown that cholesterol can suppress immune cells, regulate cell survival and modulate cancer stem cells. ${ }^{20,21}$ By summarizing the major findings of recent preclinical and clinical studies on cholesterol metabolism in immunotherapy, this review aimed to explore the potential effects of cholesterol metabolism on immunotherapy. We present the following article in accordance with the narrative review reporting checklist.

\section{Cholesterol Metabolism in the Tumor Microenvironment Affects the Function of Tumor-Infiltrating Immune Cells}

The tumor microenvironment is composed of tumor cells, local stromal cells, blood vessels, infiltrating immune cells and other related tissue cells. ${ }^{22}$ Different tumor microenvironments are formed during the development of tumors and have different beneficial or adverse influences on the occurrence of tumors. $^{23,24}$ Tumor-infiltrating immune cells, such as macrophages, lymphocytes, and neutrophils, can be altered to enhance tumor cell invasion and metastasis, angiogenesis, and immune escape. ${ }^{25}$

In the tumor microenvironment, some tumor-derived molecules have been reported to inhibit different kinds of immune cells, thereby inhibiting tumor-specific immune response. $^{26}$ Indeed, cholesterol and products of cholesterol metabolites, such as oxysterols and cholesterol ester produced by tumor cells, have been investigated in different tumor models. Cholesterol metabolism has been confirmed to affect the phenotype and ability of different types of cells that form the microenvironment, especially tumorinfiltrating immune cells (see Figure 1). ${ }^{27}$

\section{Tumor-Derived Cholesterol}

Cholesterol released by tumor cells has been proved to induce a dysfunctional state of CD8+ T cells with loss of antitumor function by overexpressing inhibitory receptors. $^{28} \mathrm{CD} 8+\mathrm{T}$ cells in the dysfunctional state in cholesterol-rich tumor tissues are positively correlated with the up-regulated expression of PD-1, 2B4 (CD244, SLAM4), T cell immunoglobulin-3 (TIM-3) and lymphocyte-activation gene 3 (LAG3). ${ }^{19}$ Further research concludes that cholesterol increases endoplasmic reticulum (ER) stress in CD8+ $\mathrm{T}$ cells and the ER stress sensor $\mathrm{x}$-box binding protein 1 (XBP1) was activated which regulated PD-1 and 2B4 transcription. ${ }^{19}$ Besides, CD8+ $\mathrm{T}$ cells can differentiate into different subsets under various cytokines. $^{29}$ In these subsets, the cytotoxic T 9 (Tc9) cells have a stronger antitumor effect than cytotoxic $\mathrm{T} 1$ (Tc1) cells. ${ }^{30}$ However, cholesterol has been shown to inhibit the antitumor function of Tc9 cells by activating the LXR signaling pathway and lowering (interleukin-9) IL-9 expression. ${ }^{30}$ To sum up, cholesterol regulation can be an effective method to upregulate the anti-tumor effect of $\mathrm{T}$ cells.

\section{Tumor-Derived Oxysterols}

Liver X receptors (LXRs), a member of the nuclear receptors, are transcription factors that can be activated by ligands. ${ }^{31,32}$ Oxysterols, such as 20(S) -hydroxylcholesterols (20HC), 24 (S) -hydroxylcholesterols (24HC), and 27- hydroxylcholesterols $(27 \mathrm{HC})$, are oxidized cholesterol metabolites that have been proved to be natural ligands of LXRs both in vitro and in vivo. ${ }^{33,34}$ The in-depth analyses of the LXR ligands/oxysterols axis have demonstrated their functions in cholesterol metabolism and associations with the pathological state, such as coronary heart disease and cerebrovascular disease. ${ }^{35,36}$ In addition, oxysterol is also associated with proliferation, migration and apoptosis of tumor cells. ${ }^{34}$

Oxysterols have been reported to influence the therapeutic effects of immunotherapy in experimental mice. ${ }^{37}$ Cancer cells release oxysterols and inhibit the expression of CC chemokine receptor type 7 (CCR7) in mature dendritic cells (DCs), thereby inhibiting the migration of DCs to draining lymph nodes and anti-tumor immune response. ${ }^{38}$ According to Carpenter et al, triple-negative breast cancer (TNBC)-produced oxysterols can inhibit the activation of macrophage and promote M2 polarization of macrophages by binding to LXR in immune cells in the tumor microenvironment. ${ }^{39}$ Moresco and colleagues have also found that tumor-derived oxysterols promoted the growth of 4T1 implanted tumor-bearing mice via creating a tumorpromoting microenvironment, especially by recruiting tumor-promoting neutrophils. ${ }^{40}$ As a member of the 


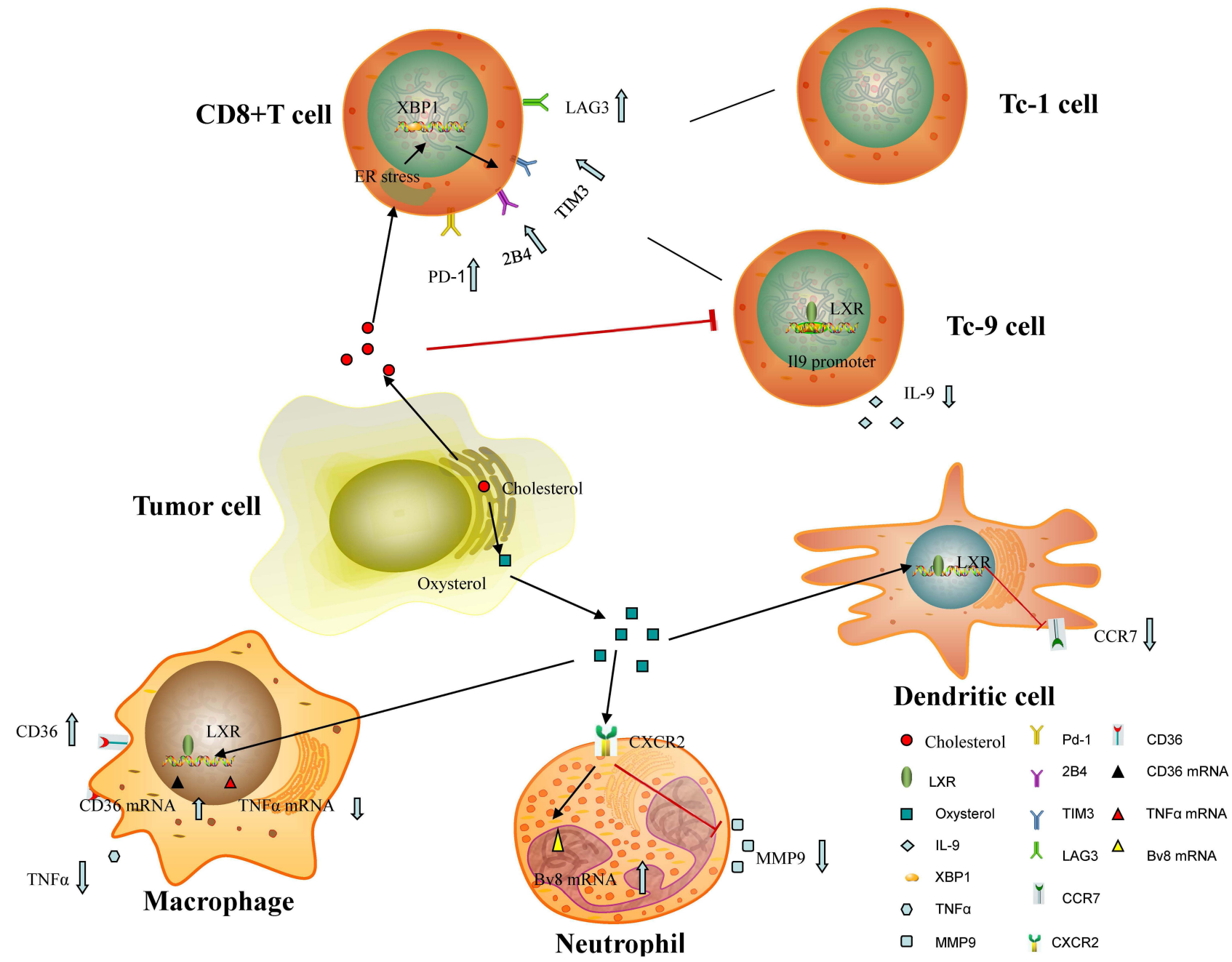

Figure I Cholesterol metabolism in the tumor microenvironment affect the function of tumor-infiltrating immune cells: Tumor-derived cholesterol can induce a dysfunctional state of CD8+ T cells with loss of antitumor function by overexpressing inhibitory receptors, inhibit the antitumor function of Tc9 cells by activating the LXR signaling pathway and lowering IL-9 expression. Tumor-derived oxysterols can reduce the expression of CCR7 in mature DCs and ultimately inhibit the migration of DCs to draining lymph nodes and anti-tumor immune response. In addition, tumor-produced oxysterols promote the recruitment of neutrophils by binding and activating CXCR2 and recruited neutrophils have been shown to promote tumor growth by releasing large amounts of total MMP-9 and enhance mRNA levels of Bv8. What's more, oxysterols released by cancer cells can inhibit the activation of macrophage and promote $M 2$ polarization of macrophages by binding to $L X R$ in immune cells.

Abbreviations: XBPI, x-box binding protein I; LAG3, lymphocyte-activation gene 3; TIM-3, T cell immunoglobulin-3; PD-I, programmed death- I; Tc9, cytotoxic T 9 cells; ER, endoplasmic reticulum; LXR, liver X receptor; TNF- $\alpha$, tumor necrosis factor $\alpha$; CXCR-2, CXC receptor 2; MMP-9, matrix metalloprotein-9; CCR-7, CC chemokine receptor type 7 .

oxysterols, 27HC inhibits CD8+ T cell activity and creates an immunosuppressive microenvironment through a complex and mostly unknown mechanism. ${ }^{41}$ Furthermore, another study has suggested that tumorderived oxysterols in the microenvironment promote the recruitment of neutrophils by binding and activating CXC receptor 2 (CXCR2) in an LXR-independent manner. ${ }^{42}$ Recruited neutrophils have been shown to release large amounts of total matrix metalloprotein-9 (MMP-9) and enhance mRNA levels of the prokineticins PK1 and PK2 (Bv8), contributing to neoangiogenesis and immunosuppression and ultimately promoting tumor growth. ${ }^{42}$ But now, the roles of the LXR/oxysterol axis in facilitating tumor invasion and metastasis by immune cells need further study. Altogether, these findings imply that the inhibition of the synthesis of cholesterol or oxysterol is a potential way to improve immunotherapy effectiveness.

\section{Intracellular Cholesterol Homeostasis is an Important Regulator of Immune Cell Function} Cholesterol, an important component of cell membranes, is therefore obviously needed for cell reproduction. ${ }^{43}$ 
Cholesterol in the free state is an important part of lipid rafts. ${ }^{44} \mathrm{~T}$ cell receptors (TCRs) reside in lipid rafts, thus cholesterol homeostasis in the plasma membrane can affect T cell function by changing TCRs. ${ }^{45}$ Cholesterol metabolism can regulate the function of immune cells by controlling a variety of immunobiological activities. ${ }^{46}$ Newly activated CD8 T cells increase cholesterol biosynthetic and input and decrease outflow via the action of sterol regulatory element binding protein (SREBP) and LXR transcription factors. ${ }^{46}$ In addition, intracellular cholesterol metabolism can also affect the activation and proliferation of macrophages and neutrophils by enriching cholesterol in the lipid raft. ${ }^{47}$

Additionally, it was reported that cholesterol esterification genes, specifically acetyl-coA acetyltransferase 1 (ACAT1), were significantly downregulated in activated CD8 T cells. ${ }^{48}$ ACAT1 is involved in an limiting step in the cholesterol esterification pathway, converting cholesterol into cholesterol ester for storage. ${ }^{49}$ Previous studies have shown that ACAT1 is abnormally expressed in some tumors, such as breast, pancreatic, and colon cancers. ${ }^{50,51}$ It has been reported that the downregulation of ACAT1 can inhibit the synthesis of cholesterol esters, thus activating CD8+ T cells. ${ }^{48,52}$

Major histocompatibility complex class II (MHC-II) which is located on the surface of antigen-presenting cells (APCs) can bind peptides derived from exogenous antigens, which is important for adaptive immune response. ${ }^{8,53}$ The association of MHC II and lipid raft is indispensable for effective T cell stimulation. ${ }^{54}$ A study suggests that cholesterol seems to affect adaptive immune response via changing MHC II function. ${ }^{55}$ Therefore, cholesterol is essential for the host's immune response, and drugs that inhibit cholesterol synthesis may reduce the immune response (see Figure 2).

\section{Drugs That Act on Cholesterol Metabolism Affect the Efficacy of Immunotherapy}

Recent studies have provided insights into the efficacy and safety of drugs primarily used to lower blood cholesterol levels, where these drugs act as immune adjuvants either alone or in combination with immunotherapy in mouse tumor models. ${ }^{56}$ The characteristics of drugs that act on cholesterol metabolism which can affect the efficacy of immunotherapy are listed in Table 1.

\section{Cholesterol-Lowering Drug}

Statins are drugs that inhibit the reductase of 3-hydroxy 3-methylglutaryl coenzyme A (HMG-CoA), which is a vital rate-limiting enzyme in the cholesterol de novo synthesis. ${ }^{57}$ Statins also play a crucial role in preventing tumor growth by promoting tumor apoptosis, inhibiting angiogenesis, and regulating immune cell function. ${ }^{58}$

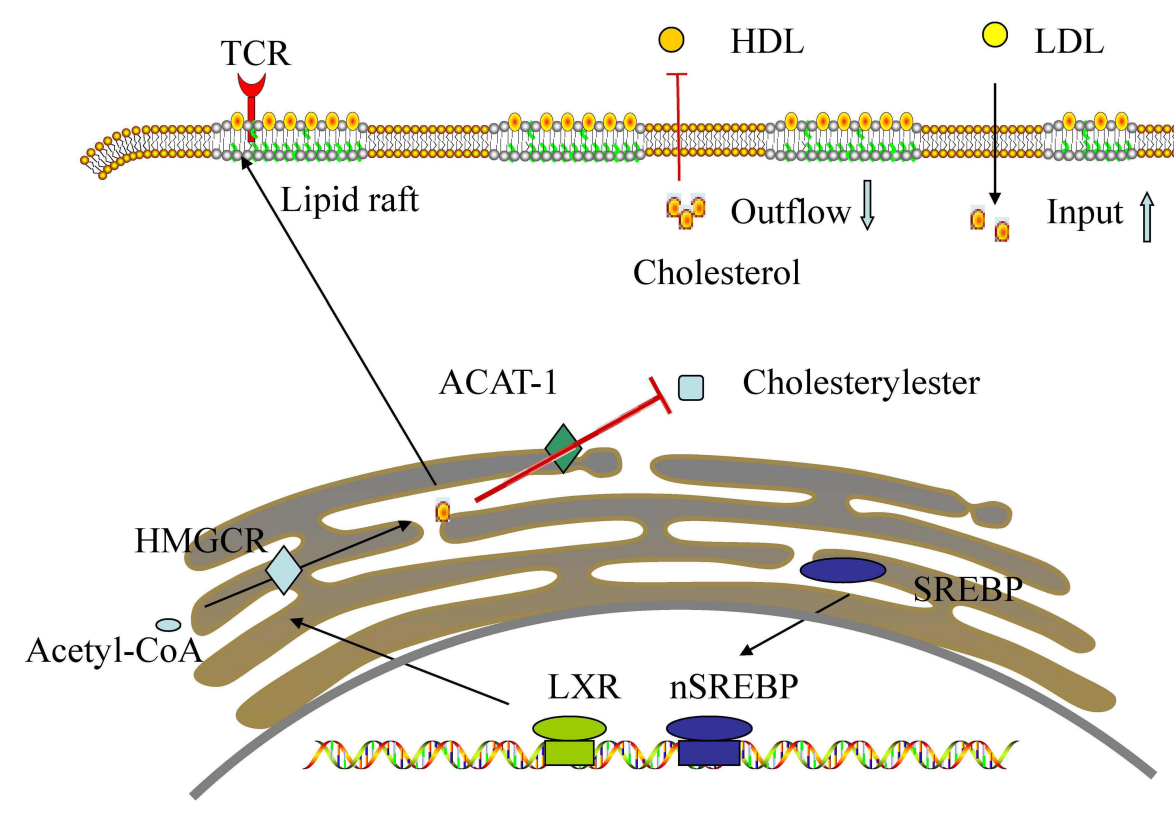

Figure 2 Cholesterol homeostasis in the activated CD8+ T cell: Newly activated CD8 T cells increase cholesterol biosynthetic and input and decrease outflow via the action of SREBP and LXR transcription factors. In addition, cholesterol esterification genes, specifically ACATI, were significantly downregulated in activated CD8 T cells. Abbreviations: TCR, T cell receptors; HDL, high-density lipoprotein; LDL, low-density lipoprotein; ACAT-I, acetyl-coA acetyltransferase I; HMGCR, 3-hydroxy-3methylglutaryl-Coenzyme $A$ reductase; LXR, liver $X$ receptor; SREBP, sterol regulatory element binding protein. 
Table I Characteristics of Drugs That Act on Cholesterol Metabolism Which Can Affect the Efficacy of Immunotherapy

\begin{tabular}{|l|l|l|}
\hline Types of Drugs & $\begin{array}{l}\text { Targets of } \\
\text { Drugs }\end{array}$ & $\begin{array}{l}\text { Representative } \\
\text { Drugs }\end{array}$ \\
\hline Statins & $\begin{array}{l}\text { HMG-CoA } \\
\text { reductase } \\
\text { PCSK9 }\end{array}$ & $\begin{array}{l}\text { Atorvastatin } \\
\text { Simvastatin } \\
\text { Evolocumab } \\
\text { Alirocumab } \\
\text { Zaragozic acids }\end{array}$ \\
$\begin{array}{l}\text { Drugs acting on LXR/ } \\
\text { oxysterol axis }\end{array}$ & $\begin{array}{l}\text { LXR/oxysterol } \\
\text { axis }\end{array}$ & Avasimibe \\
\hline
\end{tabular}

Abbreviations: HMG-CoA, 3-hydroxy 3-methylglutaryl coenzyme A; PCSK9, proprotein convertase subtilisin/Kexin type 9; LXR, liver $X$ receptor; ACATI, acetylcoA acetyltransferase I.

Statins have been reported to inhibit the growth of many cancer cell types, such as breast, prostate and pancreatic cancer. ${ }^{58,59}$ However, the sensitivity to statin-induced cell death differs among different cancer cell types. Nevertheless, there is no consensus on the influence of statins on the efficacy of ICIs.

To explore the effects of statins on immunotherapy, a prospective study with statins as a clinical factor was conducted. $^{60}$ Sixty-seven patients with mNSCLC who were treated with nivolumab were included in this study. Consequently, during nivolumab treatment, patients who received statins exhibited an increased response rate and prolonged TTF than those who did not, and the difference was statistically significant. Unfortunately, there was no significant difference in OS between the statin group and the non-statin group.

Cantini et al have conducted a prospective study to demonstrate the relationship between statins and better clinical outcome of ICIs in thoracic cancer patients. ${ }^{61}$ All enrolled patients with malignant pleural mesothelioma (MPM) or mNSCLC received ICIs therapy after first or second-line treatment failure. The results showed that the objective response rate (ORR), PFS, and OS of MPM patients using statins were improved compared with those without statins. For mNSCLC patients, the use of statins during ICIs therapy improved ORR and prolonged PFS, but not OS. Moreover, the intensity of statins is associated with improved efficacy of statins in ICIs, especially the high ones. Interestingly, this study also included 77 MPM patients receiving standard chemotherapy as a control group. In this control group, no improvement in the efficacy of chemotherapy with statins was observed, suggesting that the influence of statins on ICIs was not associated with its cholesterol-lowering effect, but with the drugs used in combination.

The influence of statins on immunotherapy needs to be confirmed by large sample studies, and the exact mechanism is not clear. Statins have been shown to extend antigen retention, strengthen antigen presentation, and activate $\mathrm{T}$ cells through the mevalonate pathway. ${ }^{62}$ Atorvastatin has been reported to affect the activated $\mathrm{T}$ cell function by inhibiting the mitogen-activated protein kinase (MAPK) and PI3K-Akt pathways activated by RAS, and the subsequent mammalian target of rapamycin (mTOR) signaling pathway promotes the overall down-regulation of inhibitory receptors, such as PD-1, CTLA-4 and lag-3. ${ }^{63}$ Moreover, statins reduce the production of inflammatory cytokines that affect immune cells and activate CD8+ $\mathrm{T}$ cells. ${ }^{64}$ Ulivieri et al also reported that simvastatin inhibited lymphocyte migration, antigen presentation, $\mathrm{T}$ cell activation, and eventually led to humoral and cellular immune damage in mice. ${ }^{65}$ Further studies are necessary to verify the role of statins as immunotherapy adjuvant. In addition to statins, proprotein convertase subtilisin/Kexin type 9 (PCSK9) inhibitors, such as evolocumab and alirocumab, are a new class of drugs that are becoming increasingly important in the treatment of hypercholesterolemia. ${ }^{66}$ PCSK9 plays a key role in cholesterol metabolism, which can regulate cholesterol levels by promoting the degradation of low-density lipoprotein receptor (LDLR) in lysosomes. ${ }^{67}$ Recent studies have shown that PCSK9 inhibitors synergistically inhibit tumor growth in mouse tumor model with anti-PD1 antibodies. ${ }^{68}$ It has been reported that the inhibition of PCSK9 can significantly increase the expression of MHC II on the surface of tumor cells, thereby promoting infiltration of cytotoxic T-cells. ${ }^{68}$

\section{Drugs Acting on LXR/Oxysterol Axis}

Zaragozic acids (ZAs), a family of fungal metabolites, are capable of blocking oxysterols formation. ${ }^{69}$ ZAs seem to affect the function of immune cells by inhibiting the formation of methotrexate and maintaining the formation integrity of isoprenoids. ${ }^{69,70}$ Studies have shown that ZAs enhanced the anti-tumor effect of immunotherapy and significantly extended the overall survival period of tumor-bearing mice, indicating that ZAs are promising anti-tumor immune response adjuvant and can be combined with immunotherapy to treat cancer patients. ${ }^{71}$

LXR/oxysterol axis has been shown to affect the activation of CD8+T cells. ${ }^{30}$ Inhibition of LXR by inverse 
agonists can lead to the migration of DC cells to lymph nodes and depress the infiltration of myeloid-derived suppressor cells (MDSCs) in the TNBC microenvironment. ${ }^{39}$ What's more, LXR inhibition is also related to the activation and proliferation of CD8+T cells and shrink of immunodependent tumors in vivo. ${ }^{39}$ LXR reverse agonist is supposed to a potential target of immunotherapy, which is expected to alter the poor efficacy of immunotherapy in TNBC patients. ${ }^{39}$

The effects of LXR agonists in multiple mouse cancer models and Phase I trials have also been explored. In contrast with the above study, the results showed that LXR agonists induced activation of $\mathrm{CD} 8+\mathrm{T}$ cells in a variety of tumor in the apolipoprotein E (ApoE) dependent manner. ${ }^{72}$ At the same time, this study also suggested that the combined application of LXR agonist and anti-PD -1 therapy produced a synergistic effect in killing tumor cells in the mouse model of lung cancer. ${ }^{72}$ In a word, LXR agitation may prevent metastasis, inhibit progression, and improve the effectiveness of ICIs therapy, especially in patients with ICIs resistance.

\section{ACATI Inhibitor}

Avasimibe is an ACAT1 inhibitor that is safe for humans and has been used to treat atherosclerosis. ${ }^{73,74}$ Recent studies have shown that avasimibe can inhibit tumor growth by damaging cell proliferation. Like other ACAT1 inhibitors, avasimibe can also enhance the effector function of $\mathrm{CD} 8+$ T cells ex vivo. ${ }^{48}$ Researchers further tested the combination of avasimibe and PD-1 inhibitors in tumor-bearing mice. ${ }^{48}$ The results showed that combination therapy was superior to monotherapy in inhibiting tumor progression and improving survival. ${ }^{48}$ It has been reported that avasimibe and anti-PD-1 play roles in cancer immunotherapy through different pathways, thus producing additive effects. ${ }^{48}$ Therefore, as a target of atherosclerosis, ACAT1 is also a potential target of cancer immunotherapy.

\section{Peripheral Blood Cholesterol Level Can Be a Biomarker to Predict the Efficacy of Immunotherapy}

Immunotherapy plays a vital role in the treatment of many kinds of tumors. However, a large number of patients remain unresponsive to immunotherapy. In addition, it is necessary to find biomarkers that can predict the efficacy of immunotherapy. ${ }^{75,76}$ The expression of PD-L1 in tumor cells is most commonly used to predict the efficacy of immunotherapy, but better predictors of response and resistance are still needed. ${ }^{77}$ At present, some peripheral blood-related biomarkers, such as neutrophil to lymphocyte ratio, C-reactive protein and lactate dehydrogenase level, have been proved to predict the efficacy of immunotherapy ${ }^{78-81}$ Qin et al evaluated the influence of blood cholesterol level on the growth of liver tumor cells in vitro models. ${ }^{82}$ They found a negative correlation between blood cholesterol levels and tumor growth. Further studies have shown that cholesterol in the blood take effect by enhancing the function of natural killer cells. ${ }^{82}$ Therefore, the cholesterol level in the blood can be a biomarker to predict the efficacy of immunotherapy.

A retrospective study collected data of metastatic nonsmall cell lung cancer (mNSCLC) patients treated with ICIs. ${ }^{83}$ Multivariate analysis confirmed that hypercholesterolemia was associated with prolonged progression-free survival (PFS) and overall survival (OS), with statistically significant differences. In a word, hypercholesterolemia has a positive impact on the prognosis of mNSCLC treated with ICIs.

Perrone and his colleagues did a retrospective study by enrolling 187 patients with metastatic tumors treated with ICIs. ${ }^{84}$ Seventy percent of the enrolled patients were NSCLC patients, and other tumor types included melanoma, urinary tract cancer and so on. Patients with high peripheral blood cholesterol levels showed longer OS and PFS than those with low plasma cholesterol. Multivariate analysis confirmed the prognostic effect of high peripheral blood cholesterol on survival. Nevertheless, the prolongation of PFS was not statistically significant. In conclusion, this study confirms that hypercholesterolemia is associated with a better prognosis in cancer patients treated with ICIs.

The reason why blood cholesterol levels can predict the efficacy of ICIs may be multifaceted and warrants further study. ${ }^{85}$ Researchers found that high plasma cholesterol levels, as a low-grade inflammatory state, might promote the proliferation and migration of tumor-associated macrophages (TAMs) and MDSCs into the tumor microenvironment, releasing many inhibitory factors and inhibiting tumor growth. ${ }^{86}$ Aguilar-Ballester et al also found that hypercholesterolemia induced the activation and proliferation of immune cells, including macrophages, neutrophils, and $\mathrm{T}$ cells. ${ }^{47}$ However, limited by small sample size, prospective studies with large sample sizes are needed to verify the predictive role of baseline blood cholesterol levels in patients with ICIs treatment. 


\section{Discussion}

Cholesterol is getting increasingly attention in cancer research because of its significant role in the prevention and treatment of cancer. ${ }^{58}$ Some retrospective studies have confirmed an association between serum cholesterol levels and the development of certain tumors, while others have found no association. ${ }^{34}$ Cholesterol in lipid rafts plays a role in maintaining the integrity of the cell membrane and assuring the signal of transmembrane receptors. ${ }^{47}$ The proliferation of cell membranes is essential not only for cancer cells, but also for immune cells. Accumulated evidence has shown that dysregulation of cholesterol metabolism plays a role in the development of cancer via affecting the function of immune cells. ${ }^{50}$ At present, the role of cholesterol metabolism in immunotherapy is poorly understood. In this review, we summarize the evidence on the association between cholesterol metabolism and immunotherapy, in order to identify new targets and biomarkers for efficacy prediction in immunotherapy.

In the tumor microenvironment, cholesterol released by tumor cells has been proven to induce a dysfunctional state of CD8+ T cells. ${ }^{28}$ In addition, cholesterol has been shown to inhibit the antitumor function of Tc9 cells, which has a stronger antitumor effect than Tc1 cells. ${ }^{29}$ Moreover, tumor-derived oxysterols can inhibit the migration of DCs to draining lymph nodes and promote the recruitment of neutrophils. ${ }^{38}$ In the meantime, recent studies show that CD8 + T cells are activated with elevated cholesterol levels in both the whole cells and cell membranes. ${ }^{46}$ Therefore, the accumulation of cholesterol can facilitate nanoclustering in T cells, ultimately promoting the antigen-presenting capacity and upregulating cholesterol synthesis and influx. Cholesterol metabolism in the tumor microenvironment suppresses immune cells; however, the accumulation of cholesterol in immune cells facilitates its activation. It has been reported that tumor-derived factors lead to intracellular accumulation of different types of oxidized-neutral lipids (triglycerides, cholesterol esters, and fatty acids) in differentiated dendritic cells, reducing the expression of MHC-type complexes on the cell surface, and thus blocking antigen cross-presentation. ${ }^{87}$ The inconsistent role of cholesterol in immune cells is not well understood. We hypothesize that tumor-derived factors in the tumor microenvironment may affect cholesterol homeostasis immune cells, thereby inhibiting the anti-tumor function. However, the function of cholesterol metabolism is complex, and this controversial conclusion requires further study.
Furthermore, several retrospective studies show the positive influence of hypercholesterolemia on the outcome of cancers treated with immunotherapy. ${ }^{83,84}$ High blood cholesterol levels in the blood are associated with prolonged PFS and OS. Obviously, cholesterol in peripheral blood has a different influence on immune cells from that in the microenvironment. Current evidence suggests that hypercholesterolemia may promote cholesterol accumulation in lipid rafts and facilitate the activation of immune cells. ${ }^{47}$ Other studies have shown that hypercholesterolemia inhibits tumor growth by improving the proliferation and migration of TAMs and MDSCs. ${ }^{86}$ Therefore, cholesterol level in peripheral blood, which is readily available, is a potential biomarker for predicting the efficacy of immunotherapy.

Statins have been shown to improve survival and reduce mortality in metastatic cancer patients. ${ }^{58}$ Due to the influence of cholesterol metabolism on immune cells, drugs that act on cholesterol metabolism may also affect the efficacy of immunotherapy. During ICIs treatment, NSCLC patients who received statins exhibited an increased response rate and longer TTF than those who did not. ${ }^{60,61}$ In other words, statins, which inhibit the synthesis of cholesterol and reduce cholesterol levels in the blood, may increase the efficacy of immunotherapy. Current studies suggest that the influence of statins on ICIs is not associated with its cholesterol-lowering effect, but with its influence on the immune system, which may explain the opposite influence of statins on immunotherapy compared to hypercholesterolemia.

\section{Conclusions}

By summarizing the major findings of recent preclinical and clinical studies on cholesterol metabolism in immunotherapy, we found that cholesterol metabolism plays an important role in regulating immune cell function and can be used as a target to enhance the efficacy of immunotherapy and a biomarker to predict the efficacy of immunotherapy.

\section{Abbreviations}

ICIs, Immune checkpoint inhibitors; PD-1, Programmed death-1; PD-L1, Programmed death ligand-1; NSCLC, Non-small cell lung cancer; CTLA-4, Cytotoxic lymphocyte antigen 4; IM-3, T cell immunoglobulin-3; LAG3, Lymphocyte-activation gene 3; BP1X-box binding protein 1; Tc9, Cytotoxic T 9 cells; Tc1, Cytotoxic T 1 cells; IL-9, Interleukin-9; LXRs, Liver X receptor; 20HC, 20(S) -hydroxylcholesterols; 24HC, 24 (S) - 
hydroxylcholesterol; 27HC, 27-hydroxylcholesterols; CCR7, CC chemokine receptor type 7; DCs, Dendritic cells; TNBC, Triple-negative breast cancer; CXCR2, CXC receptor 2; MMP-9, Matrix metalloprotein-9; Bv8, Prokineticins PK1 and PK2; TCRs, T cell receptors; SREBP, Sterol regulatory element binding protein; ACAT-1, Acetyl-coA acetyltransferase 1; MHC-II, Major histocompatibility complex class II; APCs, Antigen presenting cells; PFS, Progression-free survival; OS, Overall survival; TAMs, Tumor-associated macrophages; MDSCs, Myeloid-derived suppressor cells; HMG-CoA, 3-hydroxy 3-methylglutaryl coenzyme A; TTF, Time to treatment failure; MPM, Malignant pleural mesothelioma; MAPK, Mitogen-activated protein kinase; mTOR, Mammalian target of rapamycin; PCSK9, Proprotein convertase subtilisin/Kexin type 9; ORR, Objective response rate; ZA, Zaragozic acids; ApoE, Apolipoprotein E (ApoE).

\section{Acknowledgments}

This work was supported in part by a grant of young talents in Shanghai, National Natural Science Foundation of China (81802255), Young Talents in Shanghai (2019QNBJ), 'Dream Tutor' Outstanding Young Talents Program (fkyq1901), Clinical Research Project of Shanghai Pulmonary Hospital (fk18005), Key Discipline in 2019 (oncology), Project of Shanghai Municipal Science and Technology Commission (Project of Municipal Science and Technology Commission), Scientific research project of Shanghai Pulmonary Hospital (fkcx1903), Shanghai Municipal Commission of Health and Family Planning (2017YQ050), Innovation Training Project of SITP of Tongji University, and key projects of leading talent (19411950300). Youth project of hospital management research fund of Shanghai Hospital Association (Q1902037).

\section{Author Contributions}

All authors made substantial contributions to conception and design, acquisition of data, or analysis and interpretation of data; took part in drafting the article or revising it critically for important intellectual content; agreed to submit to the current journal; gave final approval of the version to be published; and agree to be accountable for all aspects of the work.

\section{Disclosure}

The authors declare that they have no conflicts of interest for this work.

\section{References}

1. Onoi K, Chihara Y, Uchino J, et al. Immune checkpoint inhibitors for lung cancer treatment: a review. J Clin Med. 2020;9(5):1362. doi: $10.3390 / \mathrm{jcm} 9051362$

2. He Y, Rivard CJ, Rozeboom L, et al. Lymphocyte-activation gene-3, an important immune checkpoint in cancer. Cancer Sci. 2016;107 (9):1193-1197. doi:10.1111/cas.12986

3. He Y, Cao J, Zhao C, Li X, Zhou C, Hirsch FR. TIM-3, a promising target for cancer immunotherapy. Onco Targets Ther. 2018;11:7005-7009. doi:10.2147/OTT.S170385

4. Yoshida K, Okamoto M, Sasaki J, et al. Anti-PD-1 antibody decreases tumour-infiltrating regulatory $\mathrm{T}$ cells. BMC Cancer. 2020;20(1):25. doi:10.1186/s12885-019-6499-y

5. Evrard D, Hourseau M, Couvelard A, et al. PD-L1 expression in the microenvironment and the response to checkpoint inhibitors in head and neck squamous cell carcinoma. Oncoimmunology. 2020;9 (1): 1844403 . doi: $10.1080 / 2162402 X .2020 .1844403$

6. Li B, Chan HL, Chen P. Immune checkpoint inhibitors: basics and challenges. Curr Med Chem. 2019;26(17):3009-3025. doi:10.2174/ 0929867324666170804143706

7. Herzberg B, Campo MJ, Gainor JF. Immune checkpoint inhibitors in non-small cell lung cancer. Oncologist. 2017;22(1):81-88. doi:10.1634/theoncologist.2016-0189

8. Byrne EH, Fisher DE. Immune and molecular correlates in melanoma treated with immune checkpoint blockade. Cancer. 2017;123 (S11):2143-2153. doi:10.1002/cncr.30444

9. Kyi C, Postow MA. Immune checkpoint inhibitor combinations in solid tumors: opportunities and challenges. Immunotherapy. 2016;8 (7):821-837. doi:10.2217/imt-2016-0002

10. Motzer RJ, Escudier B, McDermott DF, et al. Nivolumab versus everolimus in advanced renal-cell carcinoma. $N$ Engl $J$ Med. 2015;373(19):1803-1813. doi:10.1056/NEJMoa1510665

11. Espinosa E, Márquez-Rodas I, Soria A, et al. Predictive factors of response to immunotherapy-a review from the Spanish Melanoma Group (GEM). Ann Transl Med. 2017;5(19):389. doi:10.21037/atm.2017.08.10

12. Gounant V, Lavolé A, Quoix E. Ongoing challenges of using immunotherapy in special populations: poor performance status patients, elderly patients, and people living with HIV. Lung Cancer. 2020;145:71-75. doi:10.1016/j.lungcan.2020.04.025

13. Luo J, Yang H, Song BL. Mechanisms and regulation of cholesterol homeostasis. Nat Rev Mol Cell Biol. 2020;21(4):225-245.

14. Marquardt D, Kučerka N, Wassall SR, Harroun TA, Katsaras J. Cholesterol's location in lipid bilayers. Chem Phys Lipids. 2016;199:17-25. doi:10.1016/j.chemphyslip.2016.04.001

15. Ding X, Zhang W, Li S, Yang H. The role of cholesterol metabolism in cancer. Am J Cancer Res. 2019;9(2):219-227.

16. Murai T. Cholesterol lowering: role in cancer prevention and treatment. J Biol Chem. 2015;396(1):1-11. doi:10.1515/hsz-2014-0194

17. Heir T, Falk RS, Robsahm TE, Sandvik L, Erikssen J, Tretli S. Cholesterol and prostate cancer risk: a long-term prospective cohort study. BMC Cancer. 2016;16:643. doi:10.1186/s12885-016-2691-5

18. Cruz PM, Mo H, McConathy WJ, Sabnis N, Lacko AG. The role of cholesterol metabolism and cholesterol transport in carcinogenesis: a review of scientific findings, relevant to future cancer therapeutics. Front Pharmacol. 2013;4:119. doi:10.3389/fphar.2013.00119

19. Garcia-Estevez L, Moreno-Bueno G. Updating the role of obesity and cholesterol in breast cancer. Breast Cancer Res. 2019;21(1):35. doi:10.1186/s13058-019-1124-1

20. Lau EY, Ho NP, Lee TK. Cancer stem cells and their microenvironment: biology and therapeutic implications. Stem Cells Int. 2017;2017:3714190. doi:10.1155/2017/3714190

21. Shim SH, Sur S, Steele R, et al. Disrupting cholesterol esterification by bitter melon suppresses triple-negative breast cancer cell growth. Molecular Carcinogenesis. 2018;57(11):1599-1607. doi:10.1002/ mc. 22882 
22. Kim J, Bae JS. Tumor-associated macrophages and neutrophils in tumor microenvironment. Mediators Inflamm. 2016;2016:6058147. doi:10.1155/2016/6058147

23. Wu T, Dai Y. Tumor microenvironment and therapeutic response. Cancer Lett. 2017;387:61-68. doi:10.1016/j.canlet.2016.01.043

24. Frankel T, Lanfranca MP, Zou W. The role of tumor microenvironment in cancer immunotherapy. In: Kalinski P, editor. Tumor Immune Microenvironment in Cancer Progression and Cancer Therapy. Cham: Springer International Publishing; 2017:51-64.

25. Van Overmeire E, Laoui D, Keirsse J, Van Ginderachter JA, Sarukhan A. Mechanisms driving macrophage diversity and specialization in distinct tumor microenvironments and parallelisms with other tissues. Front Immunol. 2014;5:127.

26. Villalba M, Rathore MG, Lopez-Royuela N, Krzywinska E, Garaude J, Allende-Vega N. From tumor cell metabolism to tumor immune escape. Int J Biochem Cell Biol. 2013;45(1):106-113. doi:10.1016/j.biocel.2012.04.024

27. Raccosta L, Fontana R, Corna G, Maggioni D, Moresco M, Russo V. Cholesterol metabolites and tumor microenvironment: the road towards clinical translation. Cancer Immunol Immunother. 2016;65 (1):111-117. doi:10.1007/s00262-015-1779-0

28. Ma X, Bi E, Lu Y, et al. Cholesterol induces CD8(+) T cell exhaustion in the tumor microenvironment. Cell Metab. 2019;30(1):143156.e145. doi:10.1016/j.cmet.2019.04.002

29. Mittrücker H-W, Visekruna A, Huber M. Heterogeneity in the differentiation and function of CD8+ T cells. Arch Immunol Ther Exp. 2014;62(6):449-458. doi:10.1007/s00005-014-0293-y

30. Ma X, Bi E, Huang C, et al. Cholesterol negatively regulates IL-9-producing CD8(+) T cell differentiation and antitumor activity. $J$ Exp Med. 2018;215(6):1555-1569. doi:10.1084/jem.20171576

31. Korach-André M, Gustafsson J-Å. Liver X receptors as regulators of metabolism. Biomol Concepts. 2015;6(3):177-190. doi:10.1515/bmc2015-0007

32. Zhao C, Dahlman-Wright K. Liver $\mathrm{X}$ receptor in cholesterol metabolism. J Endocrinol. 2010;204(3):233. doi:10.1677/JOE-09-0271

33. Mutemberezi V, Guillemot-Legris O, Muccioli GG. Oxysterols: from cholesterol metabolites to key mediators. Prog Lipid Res. 2016;64:152-169.

34. Kloudova A, Guengerich FP, Soucek P. The role of oxysterols in human cancer. Trends Endrocrinol Metab. 2017;28:485-496. doi:10.1016/j.tem.2017.03.002

35. Zmysłowski A, Szterk A. Oxysterols as a biomarker in diseases. Clinica Chimica Acta. 2019;491:103-113. doi:10.1016/j. cca.2019.01.022

36. Schulman IG. Liver $X$ receptors link lipid metabolism and inflammation. FEBS Lett. 2017;591(19):2978-2991. doi:10.1002 1873-3468.12702

37. Kloudova-Spalenkova A, Holy P, Soucek P. Oxysterols in cancer management: from therapy to biomarkers. Br J Pharmacol. 2020. doi:10.1111/bph. 15273

38. Villablanca EJ, Raccosta L, Zhou D, et al. Tumor-mediated liver $\mathrm{X}$ receptor- $\alpha$ activation inhibits $\mathrm{CC}$ chemokine receptor-7 expression on dendritic cells and dampens antitumor responses. Nat Med. 2010;16(1):98-105. doi:10.1038/nm.2074

39. Carpenter KJ, Valfort AC, Steinauer N, et al. LXR-inverse agonism stimulates immune-mediated tumor destruction by enhancing CD8 T-cell activity in triple negative breast cancer. Sci Rep. 2019;9 (1):19530. doi:10.1038/s41598-019-56038-1

40. Moresco MA, Raccosta L, Corna G, et al. Enzymatic inactivation of oxysterols in breast tumor cells constraints metastasis formation by reprogramming the metastatic lung microenvironment. Front Immunol. 2018;9:2251. doi:10.3389/fimmu.2018.02251

41. Baek AE, Yu YA, He S, et al. The cholesterol metabolite 27 hydroxycholesterol facilitates breast cancer metastasis through its actions on immune cells. Nat Commun. 2017;8(1):864. doi:10.1038/s41467017-00910-z
42. Raccosta L, Fontana R, Maggioni D, et al. The oxysterol-CXCR2 axis plays a key role in the recruitment of tumor-promoting neutrophils. J Exp Med. 2013;210(9):1711-1728. doi:10.1084/ jem. 20130440

43. Chen HW, Heiniger HJ, Kandutsch AA. Relationship between sterol synthesis and DNA synthesis in phytohemagglutinin-stimulated mouse lymphocytes. Proc Natl Acad Sci U S A. 1975;72 (5):1950-1954. doi:10.1073/pnas.72.5.1950

44. Bieberich E. Sphingolipids and lipid rafts: novel concepts and methods of analysis. Chem Phys Lipids. 2018;216:114-131.

45. Molnár E, Swamy M, Holzer M, et al. Cholesterol and sphingomyelin drive ligand-independent $\mathrm{T}$-cell antigen receptor nanoclustering. $J$ Biol Chem. 2012;287(51):42664-42674. doi:10.1074/jbc. M112.386045

46. Bensinger SJ, Bradley MN, Joseph SB, et al. LXR signaling couples sterol metabolism to proliferation in the acquired immune response. Cell. 2008;134(1):97-111. doi:10.1016/j.cell.2008.04.052

47. Aguilar-Ballester M, Herrero-Cervera A, Vinué Á, Martínez-Hervás S, González-Navarro H. Impact of cholesterol metabolism in immune cell function and atherosclerosis. Nutrients. 2020;12(7):2021. doi:10.3390/nu12072021

48. Yang W, Bai Y, Xiong Y, et al. Potentiating the antitumour response of CD8(+) T cells by modulating cholesterol metabolism. Nature. 2016;531(7596):651-655. doi:10.1038/nature17412

49. Fan J, Lin R, Xia S, et al. Tetrameric acetyl-CoA acetyltransferase 1 is important for tumor growth. Mol Cell. 2016;64(5):859-874. doi:10.1016/j.molcel.2016.10.014

50. de Gonzalo-calvo D, López-Vilaró L, Nasarre L, et al. Intratumor cholesteryl ester accumulation is associated with human breast cancer proliferation and aggressive potential: a molecular and clinicopathological study. BMC Cancer. 2015;15:460. doi:10.1186/s12885-015-1469-5

51. Li J, Gu D, Lee SS, et al. Abrogating cholesterol esterification suppresses growth and metastasis of pancreatic cancer. Oncogene. 2016;35(50):6378-6388. doi:10.1038/onc.2016.168

52. Li M, Yang Y, Wei J, et al. Enhanced chemo-immunotherapy against melanoma by inhibition of cholesterol esterification in CD8 $(+) \mathrm{T}$ cells. Nanomedicine. 2018;14(8):2541-2550. doi:10.1016/j. nano.2018.08.008

53. Anderson HA, Hiltbold EM, Roche PA. Concentration of MHC class II molecules in lipid rafts facilitates antigen presentation. Nat Immunol. 2000;1(2):156-162. doi:10.1038/77842

54. Kropshofer H, Spindeldreher S, Röhn TA, et al. Tetraspan microdomains distinct from lipid rafts enrich select peptide-MHC class II complexes. Nat Immunol. 2002;3(1):61-68. doi:10.1038/ni750

55. Roy K, Ghosh M, Pal TK, Chakrabarti S, Roy S. Cholesterol lowering drug may influence cellular immune response by altering MHC II function. J Lipid Res. 2013;54(11):3106-3115. doi:10.1194/jlr.M041954

56. Blank CU, Haanen JB, Ribas A, Schumacher TN. The "cancer immunogram". Science. 2016;352(6286):658-660. doi:10.1126/ science.aaf2834

57. Sirtori CR. The pharmacology of statins. Pharmacol Res. 2014;88:3-11. doi:10.1016/j.phrs.2014.03.002

58. Hindler K, Cleeland CS, Rivera E, Collard CD. The role of statins in cancer therapy. Oncologist. 2006;11(3):306-315. doi:10.1634/theoncologist.11-3-306

59. Sassano A, Platanias LC. Statins in tumor suppression. Cancer Lett. 2008;260(1-2):11-19. doi:10.1016/j.canlet.2007.11.036

60. Omori M, Okuma Y, Hakozaki T, Hosomi Y. Statins improve survival in patients previously treated with nivolumab for advanced non-small cell lung cancer: an observational study. Mol Clin Oncol. 2019;10 (1):137-143. doi:10.3892/mco.2018.1765

61. Cantini L, Pecci F, Hurkmans DP, et al. High-intensity statins are associated with improved clinical activity of PD-1 inhibitors in malignant pleural mesothelioma and advanced non-small cell lung cancer patients. Eur J Cancer. 2021;144:41-48. doi:10.1016/j. ejca.2020.10.031 
62. Xia Y, Xie Y, Yu Z, et al. The mevalonate pathway is a druggable target for vaccine adjuvant discovery. Cell. 2018;175(4):1059-1073. doi:10.1016/j.cell.2018.08.070

63. Okoye I, Namdar A, Xu L, Crux N, Elahi S. Atorvastatin downregulates co-inhibitory receptor expression by targeting Ras-activated mTOR signalling. Oncotarget. 2017;8(58):98215-98232. doi:10.18632/oncotarget.21003

64. Côté-Daigneault J, Mehandru S, Ungaro R, Atreja A, Colombel J-F. Potential immunomodulatory effects of statins in inflammatory bowel disease. Inflamm Bowel Dis. 2016;22(3):724-732. doi:10.1097/ MIB.0000000000000640

65. Ulivieri C, Fanigliulo D, Benati D, Laghi Pasini F, Baldari CT. Simvastatin impairs humoral and cell-mediated immunity in mice by inhibiting lymphocyte homing, T-cell activation and antigen cross-presentation. Eur J Immunol. 2008;38(10):2832-2844.

66. Chen B, Shi X, Cui Y, Hou A, Zhao PA. Review of PCSK9 inhibitors and their effects on cardiovascular diseases. Curr Top Med Chem. 2019;19(20):1790-1817. doi:10.2174/1568026619666190809094203

67. Ogura M. PCSK9 inhibition in the management of familial hypercholesterolemia. $J$ Cardiol. 2018;71(1):1-7. doi:10.1016/j. jjcc.2017.07.002

68. Liu X, Bao X, Hu M, et al. Inhibition of PCSK9 potentiates immune checkpoint therapy for cancer. Nature. 2020;588(7839):693-698. doi:10.1038/s41586-020-2911-7

69. Bergstrom JD, Kurtz MM, Rew DJ, et al. Zaragozic acids: a family of fungal metabolites that are picomolar competitive inhibitors of squalene synthase. Proc Natl Acad Sci U S A. 1993;90(1):80-84. doi:10.1073/pnas.90.1.80

70. Wang Y, Metz P. A general access to zaragozic acids: total synthesis and structure elucidation of zaragozic acid D and formal syntheses of zaragozic acids A and C. Chemistry). 2011;17(12):3335-3337. doi:10.1002/chem.201003399

71. Lanterna C, Musumeci A, Raccosta L, et al. The administration of drugs inhibiting cholesterol/oxysterol synthesis is safe and increases the efficacy of immunotherapeutic regimens in tumor-bearing mice. Cancer Immunol Immunother. 2016;65(11):1303-1315. doi:10.1007/ s00262-016-1884-8

72. Tavazoie MF, Pollack I, Tanqueco R, et al. LXR/ApoE activation restricts innate immune suppression in cancer. Cell. 2018;172 (4):825-840.e818. doi:10.1016/j.cell.2017.12.026

73. Tardif J-C, Grégoire J, L'Allier PL, et al. Effects of the acyl coenzyme A: cholesterol acyltransferase inhibitor avasimibe on human atherosclerotic lesions. Circulation. 2004;110(21):3372-3377. doi:10.1161/01.CIR.0000147777.12010.EF

74. Giovannoni MP, Piaz VD, Vergelli C, Barlocco D. Selective ACAT inhibitors as promising antihyperlipidemic, antiatherosclerotic and anti-alzheimer drugs. Mini Rev Med Chem. 2003;3(6):576-584. doi:10.2174/1389557033487890

75. Gibney GT, Weiner LM, Atkins MB. Predictive biomarkers for checkpoint inhibitor-based immunotherapy. Lancet Oncol. 2016;17 (12):e542-e551. doi:10.1016/S1470-2045(16)30406-5
76. He Y, Zhang X, Jia K, et al. OX40 and OX40L protein expression of tumor infiltrating lymphocytes in non-small cell lung cancer and its role in clinical outcome and relationships with other immune biomarkers. Transl Lung Cancer Res. 2019;8(4):352-366. doi:10.21037/tlcr.2019.08.15

77. Brody R, Zhang Y, Ballas M, et al. PD-L1 expression in advanced NSCLC: insights into risk stratification and treatment selection from a systematic literature review. Lung Cancer. 2017;112:200-215. doi:10.1016/j.lungcan.2017.08.005

78. Moreira A, Leisgang W, Schuler G, Heinzerling L. Eosinophilic count as a biomarker for prognosis of melanoma patients and its importance in the response to immunotherapy. Immunotherapy. 2017;9(2):115-121. doi:10.2217/imt-2016-0138

79. Martens A, Wistuba-Hamprecht K, Geukes Foppen M, et al. Baseline peripheral blood biomarkers associated with clinical outcome of advanced melanoma patients treated with ipilimumab. Clin Cancer Res. 2016;22(12):2908-2918. doi:10.1158/1078-0432.CCR-15-2412

80. Chu X, Zhao J, Zhou J, et al. Association of baseline peripheral-blood eosinophil count with immune checkpoint inhibitor-related pneumonitis and clinical outcomes in patients with non-small cell lung cancer receiving immune checkpoint inhibitors. Lung Cancer. 2020;150:76-82. doi:10.1016/j.lungcan.2020.08.015

81. Brueckl WM, Ficker JH, Zeitler G. Clinically relevant prognostic and predictive markers for immune-checkpoint-inhibitor (ICI) therapy in non-small cell lung cancer (NSCLC). BMC Cancer. 2020;20(1):1185. doi:10.1186/s12885-020-07690-8

82. Qin WH, Yang Z-S, Li M, et al. High serum levels of cholesterol increase antitumor functions of nature killer cells and reduce growth of liver tumors in mice. Gastroenterology. 2020;158(6):1713-1727. doi:10.1053/j.gastro.2020.01.028

83. Galli G, Corsetto P, Ferrara R, et al. Impact of cholesterolemia and body mass index on outcome of metastatic non small cell lung cancer treated with immunotherapy. J Clin Oncol. 2019;37:e20691-e20691. doi:10.1200/JCO.2019.37.15_suppl.e20691

84. Perrone F, Minari R, Bersanelli M, et al. The prognostic role of high blood cholesterol in advanced cancer patients treated with immune checkpoint inhibitors. $J$ Immunother. 2020;43(6):196-203. doi:10.1097/CJI.0000000000000321

85. Bersanelli M, Cortellini A, Buti S. The interplay between cholesterol (and other metabolic conditions) and immune-checkpoint immunotherapy: shifting the concept from the "inflamed tumor" to the "inflamed patient". Hum Vaccin Immunother. 2021;1-5. doi:10.1080/21645515.2021.1902723

86. McDermott DF, Huseni MA, Atkins MB, et al. Clinical activity and molecular correlates of response to atezolizumab alone or in combination with bevacizumab versus sunitinib in renal cell carcinoma. Nat Med. 2018;24(6):749-757. doi:10.1038/s41591-018-0053-3

87. Ramakrishnan R, Tyurin VA, Veglia F, et al. Oxidized lipids block antigen cross-presentation by dendritic cells in cancer. J Immunol. 2014;192(6):2920-2931. doi:10.4049/jimmunol.1302801
OncoTargets and Therapy

\section{Publish your work in this journal}

OncoTargets and Therapy is an international, peer-reviewed, open access journal focusing on the pathological basis of all cancers, potential targets for therapy and treatment protocols employed to improve the management of cancer patients. The journal also focuses on the impact of management programs and new therapeutic agents and protocols on patient perspectives such as quality of life, adherence and satisfaction. The manuscript management system is completely online and includes a very quick and fair peer-review system, which is all easy to use. Visit http://www.dovepress.com/ testimonials.php to read real quotes from published authors. 